\title{
Patients with Klippel-Trenaunay syndrome
}

\author{
Zavacka $\mathrm{M}^{1}$, Pobehova $\mathrm{J}^{1}$, Gibarti $\mathrm{K}^{2}$, Frankovicova $\mathrm{M}^{1}$ \\ Clinic of Vascular Surgery, Faculty of Medicine UPJS and East Slovak Institute of Cardiovascular \\ Diseases, Inc., Kosice, Slovakia. cievne@vusch.sk
}

\begin{abstract}
Klippel-Trenaunay syndrome is the most frequent systemic venous angiodysplasia. It is characterized by cutaneous capillary malformations - naevus flammeus, excessive growth of soft and bone tissue, venous and lymphatic malformations. Investigative methods include: clinical examinations, venography as the evidence of dysplastic changes of superficial and / or deep venous system, the Moyne obstruction and venous insufficiency perforator. Treatment is conservative, related to that of chronic venous disease. The surgery is aimed at removing the varices and insufficient perforators (Fig. 9, Ref. 8). Text in PDF www.elis.sk.
\end{abstract}

KEY WORDS: Klippel-Trenaunay syndrome, varices, naevus flammeus, tissue hypertrophy.

\section{Clinical study 1}

39-years old woman patient was admitted to the Clinic of vascular surgery, East Slovak Institute of Cardiovascular Diseases, Inc. in Kosice for persistent swelling of the left tibia, progression of relapsing varicose veins of the left tibia with the tendency to deterioration. In anamnesis she overcame usual childhood diseases, surgery of varicose veins in the left lower limb at the local surgical department, in the dispensary of angiologist and orthopaedist for spine scoliosis. For a long time she used venotonics and anticoagulant therapy with low molecular heparin (LMWH).

Within the family there were no problems with varicose veins. At admission, on the shin on the right there were extensive stem varicosities, pre-tibial and perimalleolar oedema.

Pulsations were bilaterally well tangible at full range, trophic changes of the skin and adnexes were not present. In the sacral region there was haemangioma of the size of $10 \times 10 \mathrm{~cm}$ (Fig. 1).

The whole left lower limb was thicker by $3-4 \mathrm{~cm}$. The left foot was significantly smaller than the right one, with skeletal malformation (Figs 2 and 3).

In the control ultrasound of the lower limb vein system the finding on the left lower limb was following: $v$. saphena magna as well as $v$. saphena parva were extirpated, without any signs of recurrence, perforators on the tibia were sufficient. On the dorsal side of the thigh the cranial extension protruding from the stump of the extirpated $v$. saphena parva was confirmed by examination. It was significantly dilated with the luminal diameter of $13.5 \mathrm{~mm}$.

${ }^{1}$ Clinic of Vascular Surgery, Faculty of Medicine UPJS and East Slovak Institute of Cardiovascular Diseases, Inc., Kosice, Slovakia, and ${ }^{2}$ Clinic of Radiodiagnostics and image techniques, L. Pasteur University hospital, Kosice, Slovakia

Address for correspondence: M. Zavacka, MD, Clinic of Vascular Surgery, Faculty of Medicine UPJŠ and East Slovak Institute of Cardiovascular Diseases, Inc., Ondavska 8, SK-040 11 Kosice, Slovakia.

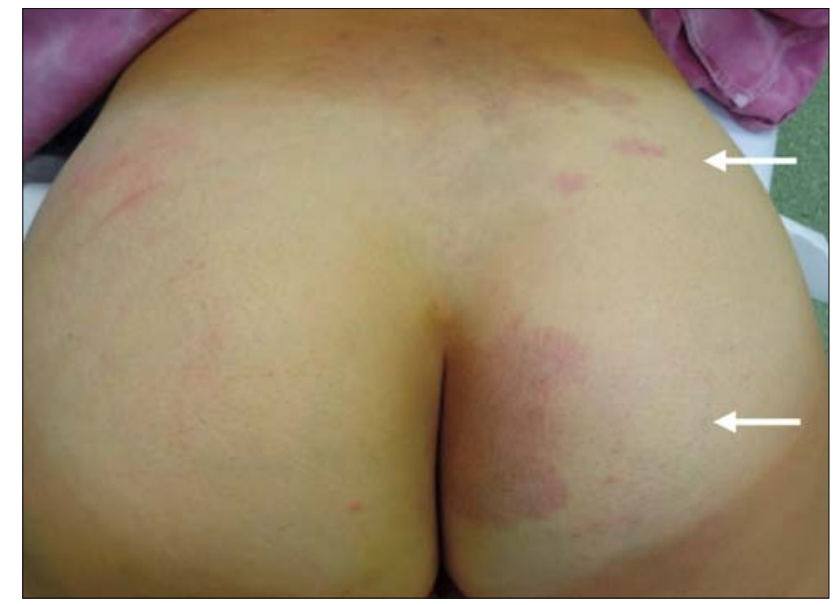

Fig. 1. Naevus flammeus in the sacral and right gluteal region.

Extension was drained by dilated perforator in the middle of dorsomedial side of the thigh running into $v$. femoralis l.sin.

In the preoperative examination the normochromatic anaemic syndrome of slight degree v.s. sideropenic, leucopaenia of slight degree, and slight hypercholesterolemia dominated in the laboratory picture. According to the examination conclusion, the patient was cardio-pulmonary compensated without any contraindications to surgery.

Under the total anaesthesia in the position on the abdomen, the stump of $v$. saphena parva l.sin was anastomized at its distal and proximal extension. The distal extension was fibrotically changed, probably after previous surgery. The proximal one was about $0.7 \mathrm{~cm}$, fibrotically changed after previous inflammations. After its skeletinization in the middle third of the back half of the thigh we ligated its tertiary part in the confluence into $v$. femoralis l.sin. This eliminated the reflux between the proximal extension of $v$. saphena parva and $v$. femoralis l.sin. 


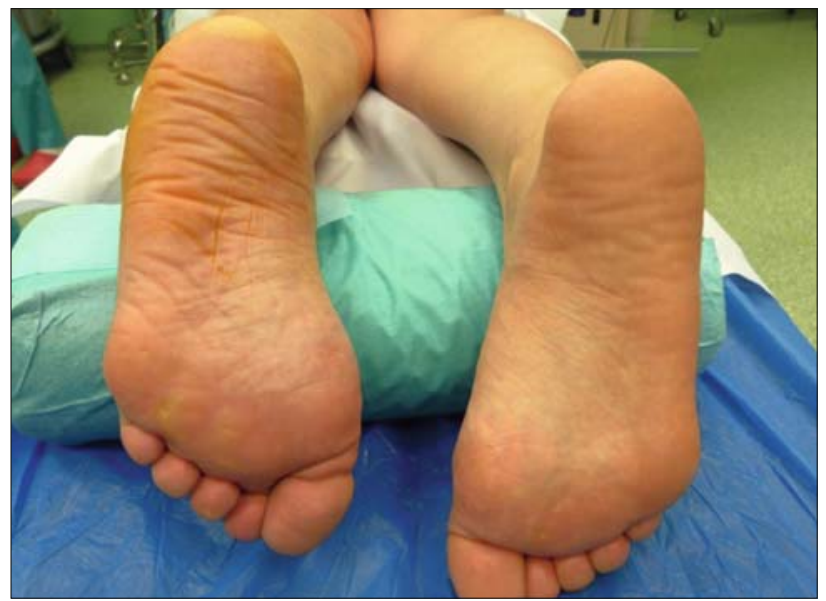

Fig. 2. Shorter left foot in the patient.

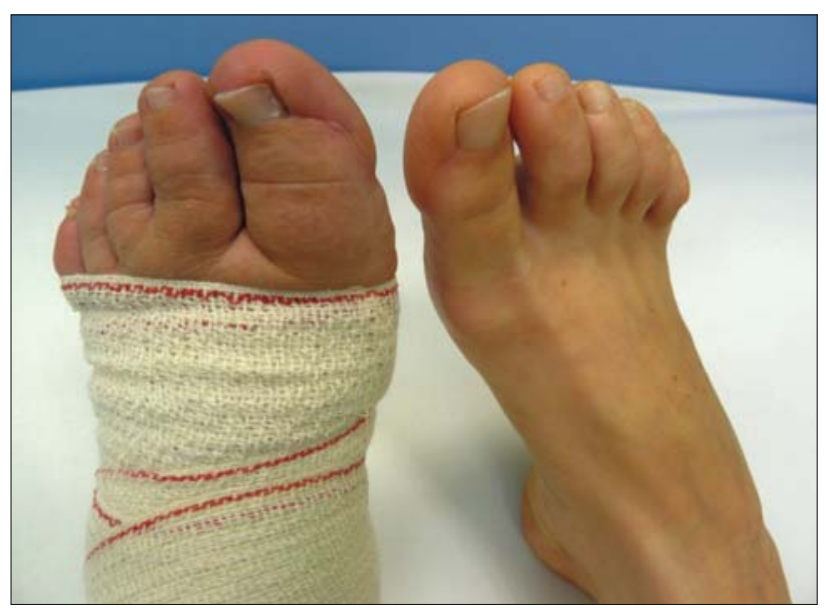

Fig. 3. Deformities and changed configuration of the left foot.

In the post-operative period the patient was afebrile, stabilised, and compensated. Surgical wounds healed p.p.i., they did not leak immediately after operation, on the left lower limb, there was high elastic bandage, Twelve hours after operation the patient was risen to her feet, rehabilitated, instructed about the necessity of high elastic bandage in home care during 4-6 weeks. On day 3 after operation she was in good condition and was released from hospital to home care

\section{Clinical study 2}

20-years old patient was admitted to the Clinic of vascular surgery, East Slovak Institute of Cardiovascular Diseases, Inc. in Kosice for the first time in December 2014 for surgical solving of the chronic venous insufficiency on the left lower limb with the presence of pronounced stem varices, concentrated mainly medially on the right shin (Fig. 4).

At admission the limb oedema was present $+2 \mathrm{~cm}$ in full size. At physical examination we observed on the chest centrally not clearly limited haemangioma of the size of 7 x $5 \mathrm{~cm}$ (Fig. 5). The
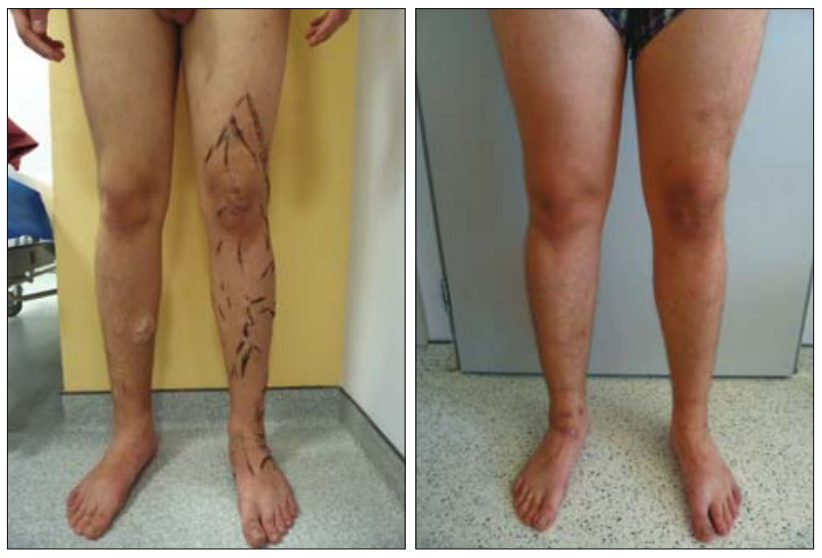

Fig. 4. Varicosity on the left lower limb in the patient with TKS (status before operation, and after operation).

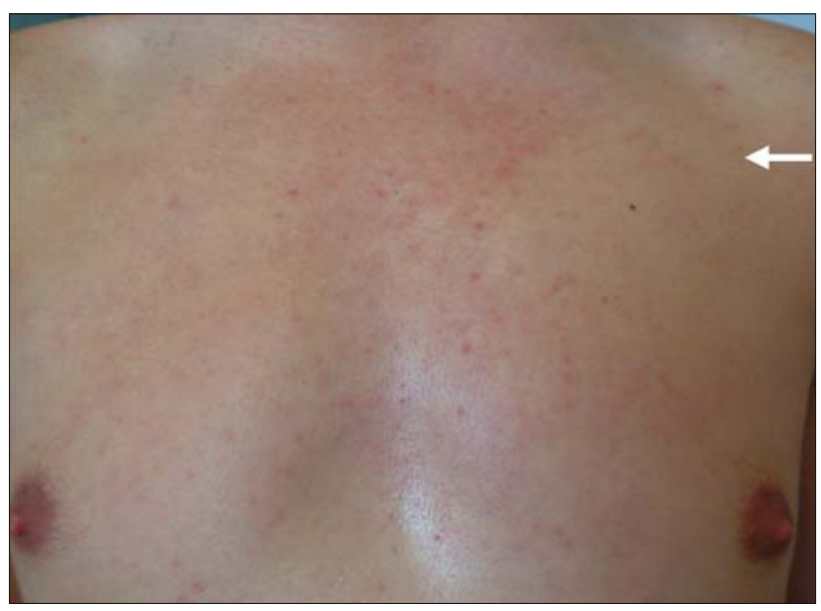

Fig. 5. Naevus flammeus localised on the chest.

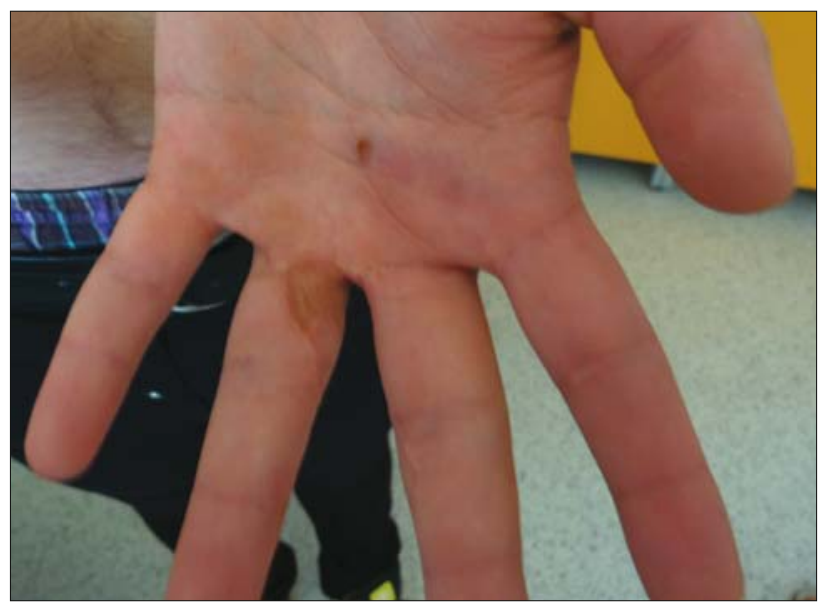

Fig. 6. Syndactyly (status post operation) in the patient with KTS.

patient in his history told about exercise-induced redness of the skin in the exposed area, deviation of the nasal septum, and status post operation of 3rd and 4th finger of the left upper limb for syndactyly (Figs 6 and 7). 


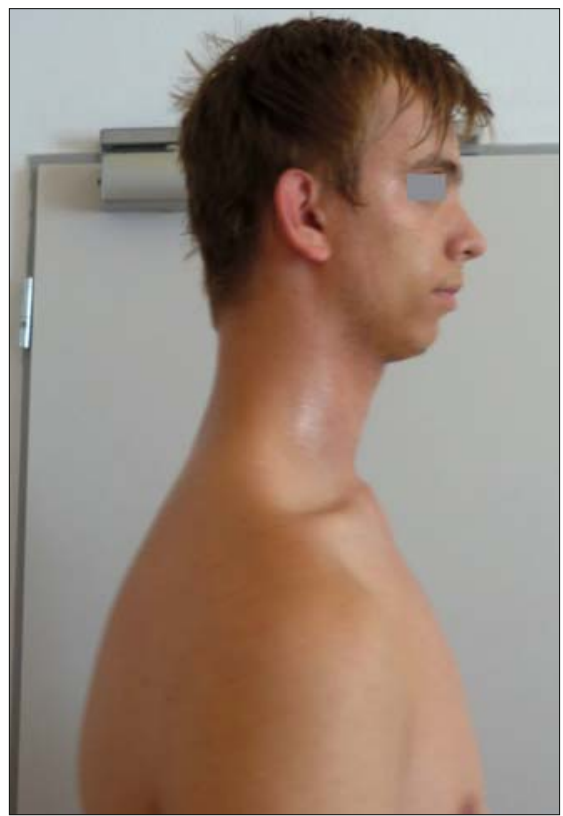

Fig. 7. Disproportion of the chest, neck, and head in the patient with KTS.

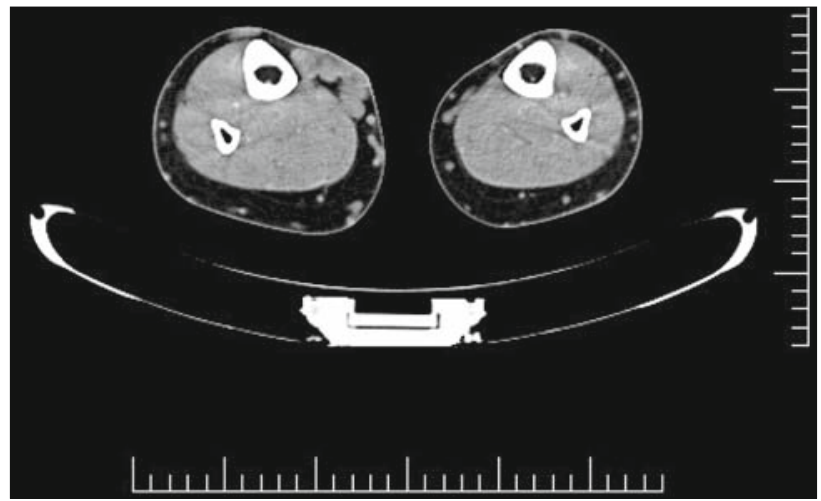

Fig. 8. Varicosity of the right tibia, strong communication of the deep and superficial venous systems (CT venography).

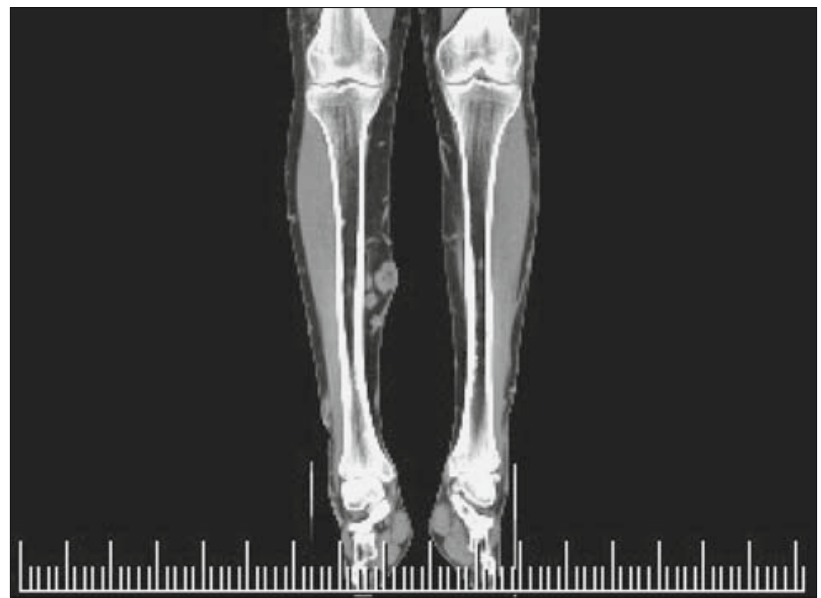

Fig. 9. Varicosity of the right tibia in the transversal section.
Subsequently, due to suspected Klippel-Trenaunay syndrome, the ultrasound and CT AG examinations of the venous system of lower limbs was supplemented. The CT AG examination showed potential A-V malformation on the lower limb in the anteromedial region of the shin, and varicose focuses throughout the whole tibia (Figs 8 and 9). The examination has not confirmed any lytic changes of bone structures. The patient was also examined in the respect of haemo-coagulation, and thrombophilia was confirmed. After diagnostics and preparation, the patient underwent an operation. The stem insufficiency VSM sin.l towards to $v$. accesoria anterior l.sin., confirmed by ultrasound and CT AG findings were the indication for surgery. Subsequently, crossectomia and stripping VSM throughout the whole course, obliterated $v v$. perforantes CI-CIII, posterior gastrocnomic and anterior lateral branch. Post operation the patient was afebrile, pressure and pulse stable and compensated, surgical wounds were calm, did not leak, healed p.p.i. The patient was informed on the next treatment procedures (elastic bandage, drinking and motion regimen), and was released to the outdoor care.

After the recovery period in February 2015 he was re-admitted to the Clinic of Vascular Surgery in order to remove SF reflux on the right lower limb, where extensive stem varices were present as well as on the collateral side.

In the early post-operative period, there were no complications, so the patient was informed about his health status and the need for the medication, and released for the outpatient care.

\section{Discussion}

Klippel and Trenaunay in 1900 described the clinical syndrome with characteristic signs on the lower limb:

1) Varices (formed already in the childhood),

2) Naevus flammeus,

3) Hypertrophy of tissues (especially bones with limb prolongation) (1).

They pointed out the venous angiodysplasia, belonging to the non-degenerative and non-inflammatory angiopathies (2). Naevus flammeus occurs in all cases. The authors also described cruciate, or dissociated type, where varices and hypertrophy occur on one and naevus on the other limb. Klippel-Trenaunay syndrome is often confused with Parks-Weber syndrome. At the other one, A-V shunt is always present, at which there are palpable whirls, pain, pulsing veins prone to rapid progression and frequent relapses. Clinically it manifests by vein dilatation and cardiac decompensation.

Vascular polyvalence at Klippel-Trenaunay syndrome can be divided into 4 types:

1) without anomaly of deep venous system, or A-V shunts;

2) with anomalies affecting deep veins (atresia, aplasia, valvular anomalies, choking fibrous bands). These can occur on one limb, or bilaterally;

3) with inactive A-V shunts;

4) with lymphatic participation (hyperplasia, hypoplasia, lymphangioma, lymph-venous fistulas)

The syndrome is often connected with malformations of lymphatic blood vessels and lymphedema, therefore it is favourable 
601-604

to perform an operation in childhood to prevent the onset of big edema and limb elongation (3).

The syndrome incidence is $1 / 20000-40000$ live-born children. The diagnosis can be made according to the specialist at the Mayo clinic if two of three main symptoms are present.

The incidence is sporadic, men and women are affected equally often, the racial predilection was not found, and symptoms may appear at birth, or during childhood (4).

Etiology of the syndrome is not clear. There are various hypotheses: Bliznak and Staple (5) supposed that intrauterine impairment of sympatic ganglia, or intermediolateral tract lead to dilatation of microscopic AV anastomoses of which malformation can develop. Servelle affirmed that blockage of the normal venous flow into deep venous system leads to the onset of venous hypertension and varix formation.

Baskerville et al (6) affirmed that the main reason of the onset is mesodermal disorder that causes persistence of microscopic $\mathrm{AV}$ anastomoses. The pathogenesis of the syndrome, as evidenced by a lot of studies, also involves genetic factors. In several patients the balanced translocation involving chromosomes 8q22.3 a 14q13 was described and found, in others translocation $t$ (5; 11), (q13.3; p15.1). Identification of two different conversions of chromosomes connected with Klippel-Trenaunay syndrome probably means the portion of more genes, which indicates its genetic heterogeneity (7).

Examination of the patient suspected of Klippel-Trenaunay syndrome involves clinical examination and venography as an evidence of dysplastic changes of the superficial, deep venous system, their obstruction, and perforator insufficiency. Angiography helps to detect $\mathrm{A}-\mathrm{V}$ fistulas, evaluate their size, frequency, and range (8).

A common problem of the followed up patients is that other symptoms connected with the development of venous insufficiency are highlighted only slowly and gradually. Practitioners often underestimate these symptoms, and so there is confusion with other vascular anomaly, capillary, or cavernous haemangioma. Therefore, the patient comes to the vascular specialist mostly in advanced stage of the disease. As well, thereno classification scale, which would determine the severity, or progression of affection at Klippel-Trenaunay syndrome (8).

The treatment is basically conservative, related to that of venous disease. The surgery is aimed at removing the varices and insufficient perforators. Stripping VSM should be done only when the deep venous system is intact. Cavernous haemangiomas in other than limb localisation can lead to more or less serious bleeding, which forces surgical treatment.

The clinical course is usually benign and slow, so adequate surgical treatment, dispensary and controls allow the affected patient normal activities (3)

In children rehabilitation is aimed at strengthening the affected limb in which hypotrophy can appear as a result of using compressive stockings.
The presence of subjective symptoms, progression of skin manifestation of venous insufficiency and venous oedema are the indication for venopharmacotherapy. The effect of venopharmaceuticals is venotic, capillary-protective, anti-inflammatory, lymphotropic, decreases blood viscosity and improves the transcutaneous partial pressure of $\mathrm{O}_{2}$. The first turn of varicose treatment is supportive by means of compressive stockings. Percutaneous injections of sclerosing substances is an alternative way of treatment for small varices. Sclerosing substances irritate the endothelium and it leads to fibrosis and obliteration of the vessel lumen (8).

\section{Conclusion}

Klippel-Trenaunay syndrome belongs to the most often occurring venous angio-dysplasias. Its incidence is confirmed at least by two of three malformations: naevus flammeus on the skin, venous and lymphatic malformation, excessive growth of soft and bone tissues.

Regarding the late diagnostics, patients usually come late in their middle age, after repeated operations on the venous system, when the right and precise diagnostics are not possible as well as the treatment of the disorders of the venous and lymphatic systems, which is documented by the case histories in our workplace.

Therefore, a general practitioner and particularly vascular specialist in case of occurrence of two symptoms of the three described should think about the potential occurrence of Klippel-Trenaunay syndrome, start correct diagnostics, and contact vascular specialist to choose an adequate type of treatment.

\section{References}

1. Klippel M, Trenaunay P. Du naevus variquex osteohypertrophique. Arch Gen Med Paris 1900; 3 : 611-672.

2. Mazuch J, Mišánik L, Mitacz K. Syndróm Klippelov-Trenaunayov. Bratisl Lek Listy 1988; 89 (12): s. 899-902.

3. Štvrtinová V et al. Choroby ciev. Bratislava: Slovak Aacdemic Press, 546-549.

4. Gloviczki P, Driscoll DJ. Klippel-Trenaunay syndrome: current management. Phlebology 2007; 22: 291-298.

5. Bliznak J, Staple TW. Radiology of angiodysplasias of the limb. Radiology 1974; 110: 35-44.

6. Baskerville PA, Ackroyd JS, Browse NL. The etiology of the Klippel-Trenaunay syndrome. Ann Surg 1985; 202: 624-627.

7. Wang Q, Timur AA, Szafranskid P et al. Identification and molecular characterization of de novo translocation $\mathrm{t}(8 ; 14)$ (q22.3;q13) associated with a vascular and tissue overgrowth syndrome. Cytogenet Cell Genet 2001; 95 (3-4): 183-186.

8. Charvátová $\mathbf{M}$ et al. Magnetická rezonance v diagnostice syndromu Klippel-Trenaunay. Ces Radiol 2013; 67 (4): 296-302.

Received November 6, 2015. Accepted June 1, 2016. 